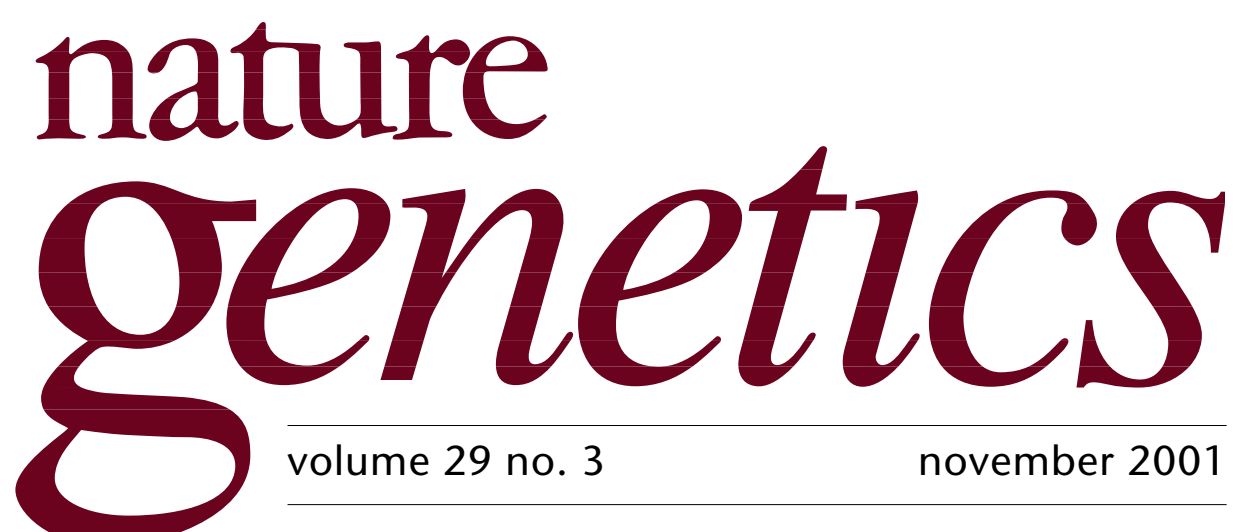

\title{
Genes, drugs and race
}

In May of this year, two studies ${ }^{1,2}$ reported the effects of heart drugs in different racial groups: in one, the drug worked equally well for blacks and whites, whereas in the second, another drug was more effective in white patients. The publications were followed by a flurry of newspaper articles with titles like "Shouldn't a pill be color blind?" or "Drug works poorly for blacks." And in the pages of the New England Journal of Medicine, where the studies were published, a heated debate continues. Critics of the studies - in particular the one showing a difference in drug response-accuse the authors of reinforcing the idea that biological differences underlie the social concept of race. Supporters of the studies say that they provide valuable medical information and a framework for identifying the molecular determinants of an individual's response to a drug.

The publications and the debate that has followed, which is not new to biomedical research, bring to light the confusion and potential harmful effects of using 'race' as a variable in medical research. Confusion because, on one hand, scientists have long been saying that at the genetic level there is more variation between two individuals in the same population than between populations and that there is no biological basis for 'race'. On the other hand, scientists continue to use race as a way to classify human biological diversity and as a substitute for biological relatedness. The potential harm to patients was highlighted in the decision by some doctors to no longer treat black patients suffering from chronic heart failure with angiotensin-converting enzyme inhibitors ${ }^{3}$, the drug that was reported to be less effective in this group of patients in one of the NEJM papers ${ }^{2}$. In practical terms, this means that because researchers found that ACE inhibitors did not seem to work in 800 patients who identified themselves as 'black', a doctor would not prescribe the drug to a patient that he or she perceives as being 'black'. And in fact some pharmaceutical companies are now exploring the concept of developing and marketing heart drugs specifically to black patients.

A paper by Wilson et al. ${ }^{4}$ (pages 265-269) in this issue of Nature Genetics shows a way to identify average differences in drug response among groups of people without any knowledge of race or ethnicity. The authors assigned individuals to four clusters by genotyping X-linked microsatellites. They found differences in the frequencies of different alleles of drug metabolizing enzymes-presumed genetic determinants of drug response-among clusters. The idea of inferring biological relatedness from multi-locus genotype data is not new, but its application to pharmacogenomic research is. Significantly, population clusters identified by genotype 
analysis seem to be more informative than those identified by skin color or selfdeclaration of race, at least in identifying variations in allele frequencies of genes encoding drug-metabolizing enzymes.

Adverse drug reactions or failure to respond to certain drugs can be influenced by polymorphisms in genes responsible for metabolizing drugs. The frequency of such polymorphisms has been found to vary between populations of common ancestry. These observations have led some researchers to readily attribute any observed differences in drug response between more or less defined ethnic or racial groups to inherent genetic differences (even if no such differences are looked for, let alone identified). The problem is that an individual's response to a drug depends on a host of factors, including overall health, lifestyle, support system, education and socioeconomic status-all of which are difficult to control for and likely to be affected, at least in the United States, by a person's 'race'.

Another problem is that race is an imprecise term. While the frequencies of certain allelic variants and mutations vary among people who share geographic origins, such ancestry is not necessarily reflected in the commonly used racial categories-American Indian, Asian, black, white, Hispanic, or other-which are based on the divisions used by the US Census Bureau ${ }^{5}$. Citing increasing diversity of the nation's population, the US Office of Management and Budget last year added the 'other' category to the Census and gave individuals the option to pick more than one race.

For this reason, the race-neutral approach proposed by Wilson et al. ${ }^{4}$ offers an attractive alternative. The authors suggest that the methodology should be used in phase III clinical trial to test for drug efficacy in different groups of patients. It is too early to determine whether the method will inform clinical practice. For one, the authors have looked at the association between population genetic structure and polymorphisms in drug-metabolizing enzymes; they have not examined response to drugs, which is likely to involve, in most cases, the interaction of several genes as well as other factors. Even then, it is unlikely that such genetic diagnostics will soon be available to most clinicians worldwide (it is still much easier to note skin color and family history). Nonetheless, the idea of replacing conventional ethnic labels with a defined genetic structure is worth pursuing, in that it moves us closer to the ultimate goal of 'individualized therapy'.

While a number of studies, including that of Wilson et al. ${ }^{4}$, have shown that average Yancy, C.W. et al.

Exner, D.V. et al. N. Engl. J. Med. 344, 1351-1357 (2001).

3. Masoudi, F.A. \& Havranek, E.P. N. Engl. J. Med. 345, 767 (2001).

Wilson, J.F. et al. Nature Genet. 29, 265-269 (2001).

5. Editorial. Nature Genet. 24, 97 (2001). differences in the genetic determinants of drug response may exist among groups, such differences are relatively small and there is considerable overlap between groups. Once all the genes that contribute to drug response are identified, doctors will be able to prescribe drugs based on patients' genotypes. For patients, this is the real promise of the Human Genome Project.

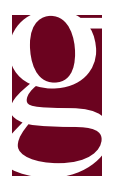

